\title{
Early Weight Loss in Exclusively Breastfed Term Neonates
}

\author{
Sahin Hamilcikan, ${ }^{1}$ Veysel Gok, ${ }^{1}$ Sultan Bent, ${ }^{1}$ and Emrah Can $^{1,}{ }^{*}$ \\ ${ }^{1}$ Department of Pediatrics, Bagcilar Training and Research Hospital, Istanbul, Turkey \\ "Corresponding author: Emrah Can, Department of Pediatrics, Bagcilar Training and Research Hospital, Istanbul, Turkey. Tel: +90-5325123606, E-mail: \\ canemrahcan@yahoo.com
}

Received 2016 October 27; Accepted 2016 December 05.

\begin{abstract}
Background: There are conflicting results about what is 'normal' weight loss in healthy term newborns and thus, when interventions, such as supplementary feeding, should be considered.

Research Aim: The current study aimed at assessing issues affecting weight loss in the early neonatal period and explaining any connections with related factors.

Methods: A prospective descriptive study was conducted on 3254 full-term, singleton newborns born at > 37 gestational weeks at a 3rd-level neonatal center in 2016.

Results: All newborns were exclusively breastfed, 758 delivered vaginally and 670 by cesarean section, making a total of 1428 newborns were weighed. Early mean weight-loss percentages were identified as $4.23 \%$ vs. $4.29 \%, 4.23 \%$ vs. $4.29 \%, 5.88 \%$ vs. $6.65 \%$, and $4.80 \%$ vs. $6.32 \%$ in Turkish and Syrian infants, respectively, measured at 12, 24, 48, and 72 hours of age. For gender evaluation, the early mean weight-loss percentage was $1.80 \%$ vs. $2.22 \%, 4.02 \%$ vs. $4.47 \%, 6.11 \%$ vs. $5.94 \%$, and $5.86 \%$ vs. $5.68 \%$, measured at $12,24,48$, and 72 hours of age. There was no difference in weight loss according to nationality or gender between the groups. For the delivery method, there was a statistical significant difference at 24 hours, 3.88 vs. 4.59, and at 30 hours, 4.07 vs. 5.58.

Conclusions: In healthy term newborns, ethnicity and gender differences did not affect weight loss. Newborns delivered vaginally had their maximal weight loss after 42 hours as opposed to 72 hours for the ones born by cesarean section. Being aware of the delayed rate of dehydration can prevent complications and unnecessary supplementation.
\end{abstract}

Keywords: Breastfeeding Experience, Breastfeeding Practices, Breastfeeding Initiation, Breastfeeding Support, Nutrition Policy

\section{Introduction}

Both the world health organization (WHO) and the American academy of pediatrics (AAP) propose breastfeeding for the first 6 months of life (1-4). Generally, healthy term neonates lose weight in their early period of life. This physiological weight loss results primarily from the distribution and loss of fluid by excreting and insensible water loss when oral intake of breast milk remains low. An infant's weight ought to be habitually and serially monitored during the period assessing breastfeeding adequacy. Breastfeeding should be started after birth as soon as possible, preferably within 30 minutes after delivery. But babies can have delayed nutrition for various reasons, such as nutrition given in certain applications, eg, water or water with sugar. A case of weight loss beyond the normal amount is a sign of poor nutritional intake or a medical problem in the neonate, thus requiring medical care and intervention. The AAP suggests prompt evaluation of the infant-mother couple if the infant loses more than $7 \%$ of its birth weight (4).

The current study aimed at assessing issues affecting weight loss in the early neonatal period (the first 3 days), and to ascertain and explain any association among the re- lated factors (gender, nationality, and delivery route difference).

\section{Methods}

Study population: A total of 3254 participants were included in a prospective descriptive study in the neonatal center from Jan 1, 2016 to Aug 31, 2016. The hospital was a 3rd-level neonatal center with a delivery number of nearly 5000 newborns per year. Neonates born by spontaneous vaginal delivery or cesarean section between gestational weeks 37 and 42, according to ultrasonographic investigations and new Ballard scoring, were included in the study. The neonates included in the study group were Turkish, while the control group included Syrian neonates.

Selection of Newborns: Instances of complicated vaginal delivery, breech presentation, multiple pregnancies, premature or postmature neonates, small- or large-forgestational-age neonates, resuscitated neonates with multiple congenital anomalies (congenital cyanotic heart diseases or respiratory distress), early sepsis, meningitis, and fetal malnutrition were excluded from the study. Fenton curves were used to process data and neonates were classified as small-for-gestational-age(SGA)( 90 percentile) 
neonates. If there was missing information on delivery method, weight or feeding, birth weight $\geq 5000 \mathrm{~g}$, multiple births, discrepancy between information sources, or no weight documented before 6 hours or before initiation of formula feeding, these cases were excluded from the study. Fetal malnutrition was defined as child and adolescent needs and strengths (CANS) scores lower or equal to 24; these neonates were included in the study (Figure 1).

Weight measurement: Weight change is defined as the change between birth weight and the weight gained subsequently, computed as a curve, as is often performed in day-to-day clinical observations. Extreme weight loss is defined as a loss of $>10 \%$ of birth weight $(5,6)$. Neonates with unexpected weight loss or weight gain values $(10 \%$ loss within the first 24 hours, $15 \%$ loss any time thenceforth; gains of $5 \%$ ) were excluded from the present study. Since the duration of recording varies by delivery method and neonates are sometimes weighed once daily following hospital birth, although sometimes not before 6 hours, weight loss curves were determined from 6 to 72 hours for cesarean delivery and from 6 to 24 hours for vaginal delivery; also, the varied accessibility of weight measurements between vaginally delivered newborns and the ones delivered by cesarean section was recorded $(7,8)$.

Statistical analysis: The statistical analysis was performed by NCSS (number cruncher statistical system) 2007. A t test was used to calculate the differences between the 2 parameters in the groups, and to compare the data, an unpaired t test and the Mann-Whitney U test were conducted. Associations were evaluated using multiple regression analysis. $\mathrm{P}<0.05$ was the level of significance.

\section{Results}

A total of 1428 newborns were included in the current prospective descriptive study, of which 758 (53.0\%) were delivered vaginally and $670(47.0 \%)$ by cesarean section. Data were available for gender, gestational age, birth weight, mean weight loss, and the number of weightmeasurement hours, summarized in Table 1 . Percentage of mean weight loss was calculated at 6,12, 18, 24, 30, 36, 42, 48, and 72 hours after birth. Groups were compared according to delivery method, gender, and nationality. Distinctions in weight loss by delivery method provided early insight and were obviously evident at intervals of 24 hours after birth. Figures 2 - 4, and Tables 2 - 4 present data regarding newborns' gender, nationality, and delivery route. Early mean weight loss percentages were identified as $4.23 \%$ vs. $4.29 \%, 4.23 \%$ vs. $4.29 \%, 5.88 \%$ vs. $6.65 \%$, and $4.80 \%$ vs. $6.32 \%$ for Turkish and Syrian infants, respectively, measured at 12 , 24,48 , and 72 hours of age. For gender evaluation, early mean weight loss percentage was $1.80 \%$ vs. $2.22 \%, 4.02 \%$ vs.
$4.47 \%, 6.11 \%$ vs. $5.94 \%$, and $5.86 \%$ vs. $5.68 \%$, measured at 12 , 24,48 , and 72 hours of age. No statistical differences were observed between the genders. For the delivery method, there was a statistical difference recorded at 24 hours, 3.88\% vs. $4.59 \%$, and at 30 hours, $4.07 \%$ vs. $5.58 \%$. Mean weight loss was firstly found to be different in the 2 delivery methods at 24 hours, continuing up to 30 hours. This difference was not observed between the genders. Multiple regression analysis was performed for each delivery method, finding that neither gender nor nationality affected early weight loss in term-breastfed neonates (Table 5).

\section{Discussion}

For several years, the generally accepted period for physiological weight loss is1 to 3 days, and in response, many neonates are biochemically evaluated or receive supplemental feeding support. Neonatal weight loss is normal after delivery with an expected loss of 5\% to $7 \%$ of birth weight. Neonates usually stop losing weight by day 5 following birth, and typically regain their birth weight by 1 to 2 weeks of age. Flaherman et al. (8) were the first to report an early weight loss nomogram for exclusively breastfed neonates.

Figure 2 shows curves for weight loss by delivery method. Mean weight loss was $4.2 \%$ at 24 hours, $7.1 \%$ at 48 hours, and $6.4 \%$ at 72 hours after birth. The maximum level of weight loss occurred at 48 hours (7.1\%). Weight gain began to rise after 48 hours for infants born vaginally. Mean weight loss was $3.8 \%$ at 24 hours, 5.8\% at 48 hours, and 5.1\% at 72 hours after birth for the vaginally delivered infants.

Bernini et al. (9) composed useful weight-loss charts in a report featuring a 5-year cohort of 1760 healthy term, exclusively breastfed, newborns in an Italian birth center, whose feeding policies followed the WHO's 10 steps to successful breastfeeding standards. An analysis of weight measurements at set time points revealed a mean weight loss of 5.95\%, with the peak occurring at 43.7 hours after birth (9). It is noteworthy that this mean weight loss was significantly lower than the $6.7 \%$ in a Portuguese study featuring a large heterogeneous cohort, which reported that infants began to gain weight earlier (10). None of the newborns in the Italian cohort lost more than $10 \%$ of their birth weight, compared to $6.1 \%$ in the Portuguese cohort (9). Macdonald et al. (11) reported the mean percentage of weight loss and also the day on which weight regain began in average as 2.7 and 8.3 days, respectively. Manganaro et al. (12) noted that infants in their study born vaginally reached their lowest weight between days 3 and 4, while infants delivered by cesarean section reached their lowest weight between days 4 and 5 . There is evidence that the 
3254 Neonate orginal cohort

865 Neonates were excluded

22 were twins, $115<2000 \mathrm{~g}$ and 58 were $5000 g, 27$ congential anomaly,

643 were miss recorded.

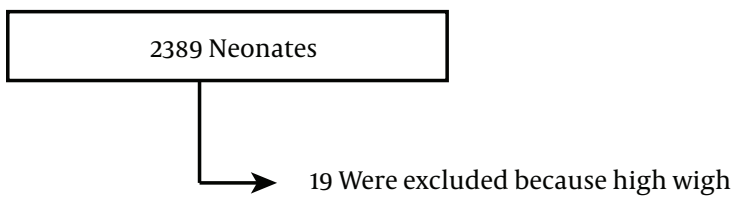

loss

942 Were not recorded wieght loss as study protocol

1428 Neonates conducted to study

1042 Turkish neonates

386 Syrian neonates

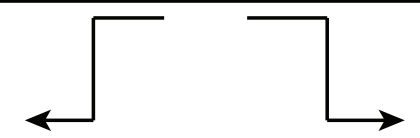

758 Vaginal born neonates

670 Cesarean born neonates

Figure 1. The Analysis of Study Participants Selection

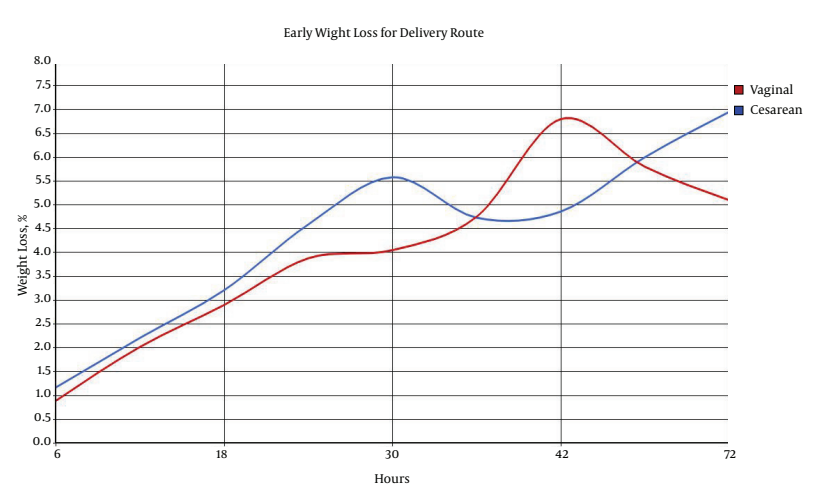

Figure 2. Early Weight Loss based on Delivery Route

initiation rates of breastfeeding are lower in infants delivered by cesarean section compared with the ones born vaginally $(13,14)$. Mean weight loss after delivery by cesarean section was $4.9 \%, 8 \%, 8.6 \%$, and $5.8 \%$ at $24,48,72$, and 96 hours, respectively. Weight gain began after 72 hours in newborns delivered by cesarean section (8). The present study observed that weight loss was statistically higher in

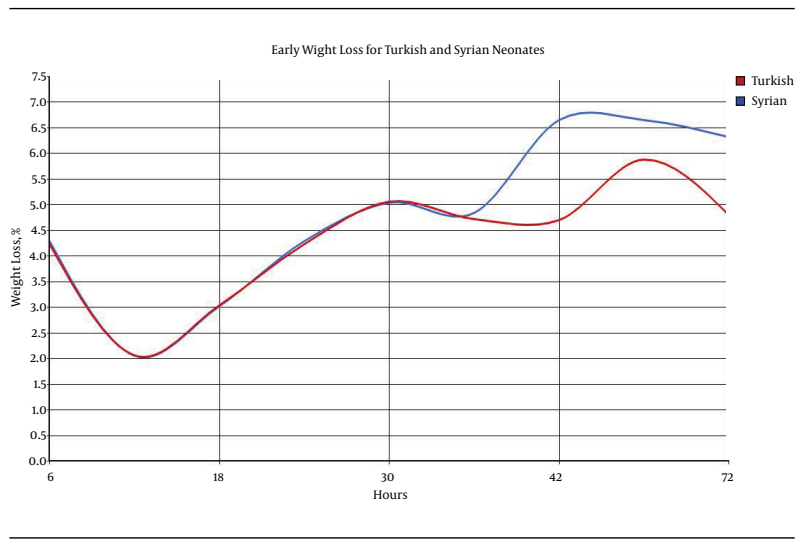

Figure 3. Early Weight Loss for Turkish and Syrian Neonates

newborns delivered by cesarean section compared with the ones born vaginally, at 24, 30, and 72 hours. Differences were not detected in other measurements. Mean weight loss in the present study was $4.59 \%, 6.0 \%$, and $6.95 \%$ at 24,48 , and 72 hours after birth in newborns delivered by cesarean section. However, the present study found no significant difference in the early-period weight loss 
Table 1. Descriptive Characteristics of Neonates ${ }^{\mathrm{a}}$

\begin{tabular}{|c|c|c|c|}
\hline & Turkis $(n=1042)$ & Syrian $(\mathbf{n}=\mathbf{3 8 6})$ & P Value \\
\hline Gender & & & 0.29 \\
\hline Male & $546(52)$ & $192(49)$ & \\
\hline Female & $496(48)$ & $194(51)$ & \\
\hline \multicolumn{4}{|l|}{ Delivery route } \\
\hline Vaginal & $610(58.5)$ & $148(38.3)$ & \\
\hline Cesarean & $432(41.5)$ & $238(61.7)$ & \\
\hline Gestational week & & & 0.001 \\
\hline 37 & $32(3)$ & $42(10.8)$ & \\
\hline 38 & $272(26.1)$ & $128(33.16)$ & \\
\hline 39 & $384(36.8)$ & $136(35.2)$ & \\
\hline 40 & $280(26.8)$ & $72(18.6)$ & \\
\hline 41 & $74(7)$ & $8(2)$ & \\
\hline Birth weight, $\mathrm{g}$ & $3301.31 \pm 460.15$ & $3202.88 \pm 429.4$ & 0.01 \\
\hline Measurement time, $h$ & & & 0.07 \\
\hline 6 & 120 & 54 & \\
\hline 12 & 118 & 58 & \\
\hline 18 & 158 & 70 & \\
\hline 24 & 354 & 128 & \\
\hline 30 & 132 & 36 & \\
\hline 36 & 28 & 10 & \\
\hline 42 & 20 & 4 & \\
\hline 48 & 112 & 22 & \\
\hline 72 & 16 & 4 & \\
\hline
\end{tabular}

${ }^{\mathrm{a}}$ Values are expressed as mean $\pm \mathrm{SD}$ or No. (\%).

Table 2. Early Weight Loss of Term Neonates, Based on Nationality ${ }^{a}$

\begin{tabular}{|c|c|c|c|c|}
\hline Early Weight Loss Based on Nationality, $\%$ & Time, $\mathbf{h}$ & Turkish $(\mathbf{n}=\mathbf{1 0 4 2})$ & Syrian $(\mathbf{n}=\mathbf{3 8 6})$ & P Value \\
\hline & 6 & $4.23 \pm 1.65$ & $4.29 \pm 3.35$ & 0.85 \\
\hline & 12 & $2.06 \pm 1.27$ & $2.06 \pm 1.05$ & 0.99 \\
\hline & 18 & $3.04 \pm 1.56$ & $3.02 \pm 1.54$ & 0.96 \\
\hline & 24 & $4.23 \pm 1.65$ & $4.29 \pm 3.35$ & 0.85 \\
\hline & 30 & $5.06 \pm 1.94$ & $5.04 \pm 2.34$ & 0.96 \\
\hline & 36 & $4.72 \pm 1.85$ & $4.84 \pm 2.56$ & 0.89 \\
\hline & 42 & $4.70 \pm 2.67$ & $6.65 \pm 1.48$ & 0.34 \\
\hline & 48 & $5.88 \pm 2.14$ & $6.65 \pm 2.11$ & 0.28 \\
\hline & 72 & $4.8 \pm 1.14$ & $6.32 \pm 1.11$ & 0.76 \\
\hline
\end{tabular}

${ }^{\mathrm{a}}$ Values are expressed as mean $\pm \mathrm{SD}$

based on the nationality. For all infants, the hospital labor ward applied the WHO's 10 steps to successful breastfeed- 
Table 3. Early Weight Loss of Term Neonates Based on Gender ${ }^{\mathrm{a}}$

\begin{tabular}{|c|c|c|c|c|}
\hline Early Weight Loss Based on Gender, \% & Time, h & Female $(n=690)$ & Male $(n=738)$ & P Value \\
\hline & 6 & $0.99 \pm 1.16$ & $1.02 \pm 0.84$ & 0.88 \\
\hline & 12 & $1.80 \pm 1.16$ & $2.22 \pm 1.27$ & 0.10 \\
\hline & 18 & $2.89 \pm 1.49$ & $3.18 \pm 1.61$ & 0.31 \\
\hline & 24 & $4.02 \pm 1.78$ & $4.47 \pm 2.57$ & 0.11 \\
\hline & 30 & $5.08 \pm 1.79$ & $5.04 \pm 2.20$ & 0.93 \\
\hline & 36 & $4.83 \pm 1.86$ & $4.63 \pm 2.22$ & 0.83 \\
\hline & 42 & $4.25 \pm 2.77$ & $6.57 \pm 0.86$ & 0.14 \\
\hline & 48 & $6.11 \pm 2.17$ & $5.94 \pm 2.14$ & 0.75 \\
\hline & 72 & $5.86 \pm 1.26$ & $5.68 \pm 2.16$ & 0.62 \\
\hline
\end{tabular}

${ }^{\mathrm{a}}$ Values are expressed as mean $\pm \mathrm{SD}$.

Table 4. Early Weight Loss of Term Neonates Based on Delivery Route ${ }^{\mathrm{a}}$

\begin{tabular}{|c|c|c|c|c|}
\hline Early Weight Loss Based on Delivery Route & Time, h & Vaginal Delivery $(n=758)$ & Cesarean Section $(n=670)$ & P Value \\
\hline & 6 & $0.89 \pm 0.88$ & $1.17 \pm 1.12$ & 0.30 \\
\hline & 12 & $2.02 \pm 1.23$ & $2.21 \pm 1.07$ & 0.52 \\
\hline & 18 & $2.90 \pm 1.66$ & $3.21 \pm 1.39$ & 0.29 \\
\hline & 24 & $3.88 \pm 1.81$ & $4.59 \pm 2.50$ & 0.01 \\
\hline & 30 & $4.05 \pm 2.19$ & $5.58 \pm 1.73$ & 0.01 \\
\hline & 36 & $4.75 \pm 1.45$ & $4.73 \pm 2.24$ & 0.98 \\
\hline & 42 & $6.80 \pm 1,02$ & $4.86 \pm 2.50$ & 0.85 \\
\hline & 48 & $5.80 \pm 1.46$ & $6.00 \pm 2.20$ & 0.15 \\
\hline & 72 & $5.10 \pm 1.14$ & $6.95 \pm 1.11$ & 0.01 \\
\hline
\end{tabular}

${ }^{\mathrm{a}}$ Values are expressed as mean $\pm \mathrm{SD}$.

Table 5. Early Weight Loss of Term Neonates Based on Different Factors

\begin{tabular}{|c|c|c|c|c|c|c|}
\hline \multirow{3}{*}{ Model } & \multicolumn{6}{|c|}{ Coefficients $^{\mathrm{a}}$} \\
\hline & & \multicolumn{2}{|c|}{ Unstandardized Coefficients } & \multirow{2}{*}{$\begin{array}{c}\text { Standardized Coefficients } \\
\text { Beta }\end{array}$} & \multirow{2}{*}{ t } & \multirow{2}{*}{$\begin{array}{l}\text { P Value } \\
\text { Std. Error }\end{array}$} \\
\hline & & B & Std. Error & & & \\
\hline \multirow[t]{4}{*}{$\mathbf{1}$} & (Constant) & 52.766 & 18.241 & & 2.893 & 0.004 \\
\hline & Nationality & 1.044 & 1.793 & 0.021 & 0.583 & 0.560 \\
\hline & D. Route & -11.779 & 1.600 & -0.271 & -7.360 & 0.000 \\
\hline & B. Weight & 0.003 & 0.002 & 0.069 & 1.872 & 0.062 \\
\hline
\end{tabular}

${ }^{a}$ Dependent variable: weight loss.

ing standards. Similar to the present study, Flaherman et al. (8) reported no difference in the early weight loss of breastfed newborns according to gender. This degree of weight loss could be related to high risk of pathological features and hypernatremic dehydration, requiring supplementation intervention, although it should be avoided since it may discourage the initiation of breastfeeding and decrease its duration $(4,15)$. Initiation of supplementation 


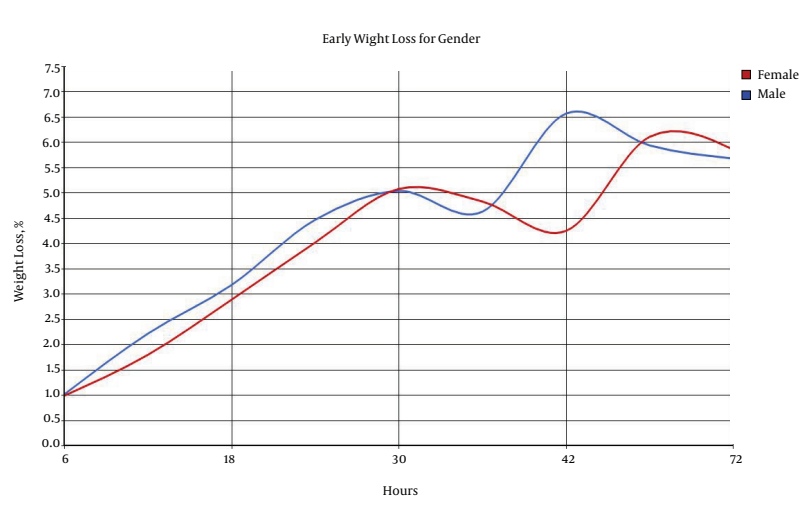

Figure 4. Early Weight Loss Based on Gender

with banked human milk or commercial infant formula is only recommended when the infant has lost more than $7 \%$ of neonatal birth weight between 5 and 10 days of age when the infant exhibits signs of dehydration and the mother's milk supply is limited (16). All newborns were exclusively breastfed in this study, no supplementation was initiated.

As a result, according to the study of healthy term newborns, ethnicity and gender differences did not affect weight loss. Depending on early birth by cesarean section or vaginal delivery, maximum weight loss in newborns occurred after 42 hours in vaginal delivery vs. 72 hours in cesarean delivery. Being aware of the daily weight loss rate of dehydration can prevent complications and unnecessary supplementation.

\section{References}

1. World Health Organization . Global Strategy for Infant and Young Child Feeding 2003. Available from: www.who.int/nutrition/ publications/infantfeeding/en/index.html.

2. U. S. Preventive Services Task Force . Primary care interventions to promote breastfeeding: U.S. Preventive Services Task Force recommendation statement. Ann Intern Med. 2008;149(8):560-4. [PubMed: 18936503].
3. Committee on Health Care for Underserved Women ACOO. ACOG Committee Opinion No. 361: Breastfeeding: maternal and infant aspects. Obstet Gynecol. 2007;109(2 Pt 1):479-80. [PubMed:17267864].

4. Section on B. Breastfeeding and the use of human milk. Pediatrics. 2012;129(3):e827-41. doi: 10.1542/peds.2011-3552. [PubMed: 22371471].

5. Chantry CJ, Nommsen-Rivers LA, Peerson JM, Cohen RJ, Dewey KG. Excess weight loss in first-born breastfed newborns relates to maternal intrapartum fluid balance. Pediatrics. 2011;127(1):e171-9. doi: 10.1542/peds.2009-2663. [PubMed: 21173007].

6. Dewey KG, Nommsen-Rivers LA, Heinig MJ, Cohen RJ. Risk factors for suboptimal infant breastfeeding behavior, delayed onset of lactation, and excess neonatal weight loss. Pediatrics. 2003;112(3 Pt 1):607-19. [PubMed: 12949292].

7. Datar A, Sood N. Impact of postpartum hospital-stay legislation on newborn length of stay, readmission, and mortality in California. Pediatrics. 2006;118(1):63-72. doi: 10.1542/peds.2005-3044. [PubMed: 16818550].

8. Flaherman VJ, Schaefer EW, Kuzniewicz MW, Li SX, Walsh EM, Paul IM. Early weight loss nomograms for exclusively breastfed newborns. Pediatrics. 2015;135(1):e16-23. doi: 10.1542/peds.2014-1532. [PubMed: 25554815].

9. Bertini G, Breschi R, Dani C. Physiological weight loss chart helps to identify high-risk infants who need breastfeeding support. Acta Paediatr. 2015;104(10):1024-7. doi: 10.1111/apa.12820. [PubMed: 25283590].

10. Fonseca MI, Severo M, Santos AC. A new approach to estimating weight change and its reference intervals during the first 96 hours of life. Acta Paediatr. 2015;104(10):1028-34. doi: 10.1111/apa.12894. [PubMed: 25488548].

11. Macdonald PD, Ross SR, Grant L, Young D. Neonatal weight loss in breast and formula fed infants. Arch Dis Child Fetal Neonatal Ed. 2003;88(6):F472-6. [PubMed:14602693].

12. Manganaro R, Mami C, Marrone T, Marseglia L, Gemelli M. Incidence of dehydration and hypernatremia in exclusively breast-fed infants. $J$ Pediatr. 2001;139(5):673-5. [PubMed:11713445].

13. Golding J, Greenwood R, Birmingham K, Mott M. Childhood cancer intramuscular vitamin $\mathrm{K}$, and pethidine given during labour. $B M J$. 1992;305(6849):341-6. [PubMed:1392886].

14. Passmore SJ, Draper G, Brownbill P, Kroll M. Case-control studies of relation between childhood cancer and neonatal vitamin K administration. BMJ. 1998;316(7126):178-84. [PubMed: 9468681].

15. Becker GE, Remmington T. Early additional food and fluids for healthy breastfed full-term infants. Cochrane Database Syst Rev. 2014(11):CD006462. doi: 10.1002/14651858.CD006462.pub3. [PubMed 25420475].

16. The Academy of Breastfeeding Medicine . Breastfeeding 2016. Available from: www.bfmed.org. 